\title{
An Enhanced Speech Emotion Recognition System Based on Discourse Information
}

\author{
Chun Chen, Mingyu You, Mingli Song, Jiajun Bu, and Jia Liu \\ College of Computer Science, YuQuan Campus, ZheJiang University \\ Hangzhou, P.R.CHINA, 310027 \\ \{chenc, roseyoumy, brooksong, bjj, liujia\}@zju.edu.cn
}

\begin{abstract}
There are certain correlation between two persons' emotional states in communication, but none of previous work has focused on it. In this paper, a novel conversation database in Chinese was collected and an emotion interaction matrix was proposed to embody the discourse information in conversation. Based on discourse information, an enhanced speech emotion recognition system was presented to improve the recognition accuracy. Some modifications were performed on traditional KNN classification, which could reduce the interruption of noise. Experiment result shows that our system makes $3 \%-5 \%$ relative improvement compared with the traditional method.
\end{abstract}

\section{Introduction}

Researches on understanding and modelling human emotions have attracted increasing attention in the artificial intelligence field. As a major indicator of human emotions, speech plays an important role in detecting affective states. Accurate emotion recognition from speech signals will benefit the human-machine interaction [1]. It has broadly potential applications in areas such as education, consumer service and entertainment.

There are lots of researches that attempt to characterize emotional states of human speech. Most previous efforts involving speech emotion recognition have tended to focus on either lexical [2, 3] or acoustic information [4, 5, 6]. These studies usually used non-natural speech, including short isolated utterances, expressed by professional actors. Systems embedding lexical features into emotion recognition assumed that certain words correlated with emotional states. But as we know, the relationship between words and emotions is fuzzy and sometimes confused. One word may indicate several possible emotions and one emotional state can be conveyed by different words. Besides, lexical information always needs manual transcription for each utterance, which is difficult to be realized automatically.

On the other hand, researches on emotion detection of spoken dialog system tried to classify more natural occurring emotion of actual users. In order to achieve better performance, lexical and acoustic feature sets were augmented with additional features such as context and discourse information $7,8,9,10$, . Contextual factors really have influences on emotion identification, which can 
be found in the work of Crystal[11,12,13]. Liscombe[7] et al. included prosodic context, lexical context and discourse context as contextual features in emotion prediction and increased classification accuracy by $2.6 \%$. Contributions made by context information indicate that emotion is expressed in a language environment. Traditional operation extracting prosodic features from isolated utterances is inconsistent with emotion perception of human beings. Contextual features focus on utterances of single person, but dialogs appear frequently in daily life. Many studies paid attention to dialog system and added discourse information into emotion recognition $8,9,10$. Ang et al. 8 included discourse features such as turn location within the conversation and dialog acts of the current turn (repeat, repair, neither). Lee et al. 9. labeled dialog acts as rejection, repeat, rephrase, ask-start over or none of the above. The addition of discourse information added approximately $3 \%$ relative improvement over using lexical and prosodic features alone. But the discourse features considered were only based on the categorization of users' responses when interacting with machine. Few work paid attention to the dialog between two persons. But it is known that the emotions of two talkers have certain correlation.

This paper presents research on simultaneously recognizing two talkers' emotional states using discourse information. In addition to extracting typical acoustic features, we combined emotional correlation between two persons in dialog into speech emotion recognition. Using this extended method, we observed an increase in prediction accuracy.

\section{Conversation Corpus and Discourse Information}

In the field of emotion recognition, there are ongoing debates concerning how to define basic emotion categories. Different researchers have different opinions and some psychologists even argue against the categorical labels for human emotions. In this paper, we just focus on the archetypal emotions - happy, sad, fear, angry, surprise and neutral. Besides, how to obtain amount of realistic data for research is another hard task. Most studies in speech emotion recognition asked subjects to simulate certain emotions with neutral semantic content [14. Since those data sets are limited to utterances of single person in archetypal emotions, results based on them may still have distance to real-life scenarios. On the other hand, it's hard for real data to cover all the emotion categories needed and the noise in real environment is also a problem. With aforementioned analysis, we should turn to sources containing conversations and happening in a relatively quiet background. The living theater and broadcast drama are suitable sources for dialog corpus in controlled environment. They also embody most emotional correlation of conversation in daily life. As the beginning of dialog corpus collection, we tried to extract 4000 conversations from Chinese drama lasting hundreds of hours totally. Our data corpus covers the six archetypal emotions mentioned above and contains dialogs covering man to man, man to woman and woman to woman.

In table 1, we mark two persons having conversation as A and B. A represents the first person and $\mathrm{B}$ denotes the second one. The left part of table 1 presents 
Table 1. Distribution of The Other's Emotion When Given One Person's Emotional State (The abbreviated emotion labels in line are same to those in column)

\begin{tabular}{c|c|c|c|c|c|c||c|c|c|c|c|c|c}
\hline A & Ne.(\%) & An. & Fe. & Ha. & Sa. & Su. & B & Ne. & An. & Fe. & Ha. & Sa. & Su. \\
\hline Neutral & 26.67 & 22.22 & 6.67 & 6.67 & 11.11 & 26.67 & Neutral & 23.53 & 27.45 & 13.73 & 11.76 & 5.88 & 17.65 \\
\hline Angry & 32.56 & 46.51 & 2.33 & 2.33 & 4.65 & 11.63 & Angry & 20.83 & 41.67 & 12.5 & 0 & 8.33 & 16.67 \\
\hline Fear & 41.18 & 35.29 & 5.88 & 0 & 11.76 & 5.88 & Fear & 50 & 16.67 & 16.67 & 16.67 & 0 & 0 \\
\hline Happy & 30 & 0 & 5 & 40 & 5 & 20 & Happy & 16.67 & 5.56 & 0 & 44.44 & 0 & 33.33 \\
\hline Sad & 20 & 26.67 & 0 & 0 & 40 & 13.33 & Sad & 29.41 & 11.76 & 11.76 & 5.88 & 35.29 & 5.88 \\
\hline Surprise & 36 & 32 & 0 & 24 & 4 & 4 & Surprise & 48 & 20 & 4 & 16 & 8 & 4 \\
\hline
\end{tabular}

A's emotion distribution when given the emotional state of B. Similarly, emotion distribution of $\mathrm{B}$ is shown at the right part of table 1 . As an example, we look into the first line of part A. This line exhibits A's emotion distribution when the emotional state of B is neutral. From the listed numbers, we find that when B is neutral, the probability of A on neutral is $26.67 \%$. Probabilities of A on angry, fear, happy, sad and surprise are $22.22 \%, 6.67 \%, 6.67 \%, 11.11 \%$ and $26.67 \%$, respectively. Different probability on each emotion category implies that B will affect the emotional state of $\mathrm{A}$ in dialog and vice versa. For example, when $\mathrm{B}$ is happy, A is likely to be also happy considering the corresponding high probability. We were wondering if we could embed this information into speech emotion recognition in order to improve the recognition accuracy. A detailed experiment is designed in the following section.

\section{An Enhanced Speech Emotion Recognition}

Based on the analysis above, we propose an enhanced speech emotion recognition system in Figure 1. The system is composed of two parts - training and

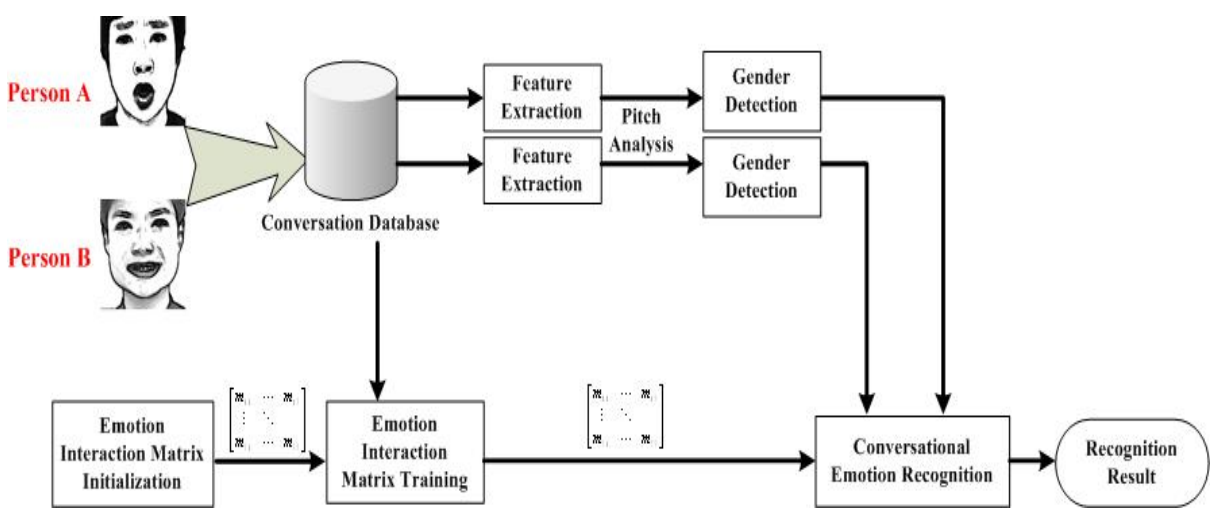

Fig. 1. System Overview 
testing. In the off-line training phase, we collected 4000 dialogs into the conversation database. The corpus includes thousands of people instead of two in Figure 1 which is just for simplification. An initialized emotion interaction matrix was trained to embody the emotion correlation between people in dialog in the training course. When new data came into the system for testing, acoustic features were extracted and gender classification was performed based on pitch analysis. Then a method combining emotion interaction matrix was proposed to recognize emotional states of both persons using those acoustic features. The whole process can be divided into three steps mentioned below.

\subsection{Acoustic Features Extraction}

The conversation corpus we collected is sampled at 16kHZ frequency and 16 bits resolution with monophonic Windows PCM format. In this study, we extracted 48 prosodic and 16 formant frequency features. Prosody is mainly related to the rhythmic aspects of speech, and believed to be the primary indicator of speakers' emotion state. The extracted prosodic features include: max, min, mean, median of Pitch (Energy); mean, median of Pitch (Energy) rising/ falling slopes; max, mean, median duration of Pitch (Energy) rising/ falling slopes; mean, median of Pitch (Energy) plateaux at maxima/ minima; max, mean, median duration of Pitch (Energy) plateaux at maxima/ minima. Here, if the first derivative is approximately zero and the second derivative is positive, the point belongs to a plateau at a local minimum. If the second derivative is negative, it belongs to a plateau at a local maximum. We also investigated formant frequency features which are widely used in speech processing applications. Statistical properties including max, min, mean, median of the first, second, third, and fourth formant were extracted.

\subsection{Dimensionality Reduction}

Because high dimensional data can dramatically raise computational complexity and decrease classification accuracy in speech emotion recognition, the 64dimensional acoustic features extracted above should be compressed. Principal Component Analysis (PCA) was employed as dimensionality reduction method in our study. PCA tends to find a t-dimensional subspace whose basis vectors correspond to the maximum variance direction in the original s-dimensional space $(t \ll s)$. Original data set is projected into the t-dimensional subspace with projection matrix $W_{P C A}$.

In the experiment, speaker independent emotion recognition was investigated because of the thousands of speakers involved in conversation database. But gender classification was still performed on speech data because of the difference of acoustic features between female and male. 10-fold cross-validation method was adopted considering the confidence of recognition results. So, $7200(90 \% * 4000 * 2)$ 64-dimensional vectors were used to train PCA. We used a $7200 * 64$ matrix X to represent these vectors. After $\mathrm{X}$ was normalized and mean-subtracted, we got matrix Y. $Y^{T} Y$ formed a covariance matrix $M$ which was $64 * 64$. Eigenvalues and 
eigenvectors were computed for M. Eigenvectors corresponding to the largest $\mathrm{t}$ eigenvalues were selected to create the PCA projection matrix $W_{P C A}$. $\mathrm{t}$ was the number of eigenvalues that guaranteed energy $\mathrm{E}$ was greater than 0.9 . Here energy E was defined in equation(1):

$$
E_{t}=\sum_{j=1}^{t} \lambda_{j} / \sum_{j=1}^{64} \lambda_{j}
$$

where $\lambda_{j}$ was the jth eigenvalue. In our experiment, t equaled to $27.3600(7200 / 2)$ training conversations and $400(800 / 2)$ testing ones were both projected into subspace using $W_{P C A}$.

\subsection{Emotion Recognition Based on Discourse Information}

Having the low dimensional features, K-Nearest-Neighbor(KNN) was adopted to classify the data into six emotional states. K-Nearest-Neighbor is a simple classification which range the testing data into the class most of its $\mathrm{k}$ nearest neighbors belonging to. It is the classical implementation of KNN. We made some modifications to KNN for the sake of our enhanced recognition system. $\mathrm{K}$ nearest neighbors were calculated for testing utterance which was same to KNN classification. In the process of K nearest neighbors' calculation, Euclidean distance was adopted as the distance measurement between feature vectors of training utterance and testing utterance. $\mathrm{K}$ nearest neighbors belonged to $M$ classes $\left\{C_{1}, C_{2}, \cdots, C_{M}\right\}$. The probability of belonging to $C_{i}$ was defined by: $P_{i}=N_{i} / N_{k}$ where $N_{i}$ denoted the number of nodes belonging to $C_{i}$ and $N_{k}$ equaled to $\mathrm{k}$ which was the total number of nodes. So the probabilities for $M$ classes was $\left\{P_{1}, P_{2}, \cdots, P_{M}\right\}$ and we used $P_{m_{1}}$ to be the highest probability and $P_{m_{2}}$ to be the second. In classical KNN, $C_{m_{1}}$ with $P_{m_{1}}$ is selected as the recognition result for test utterance. However, we'd like to make our decision based on $P_{m_{1}}$ and $P_{m_{2}}$ instead of depending on $P_{m_{1}}$ alone.

Different from conventional methods, the emotional states of two persons in dialog were recognized together in our system. Let us use $M_{A}$ to stand for the emotion interaction matrix of $\mathrm{A}$ when given emotional state of $\mathrm{B}$ and $M_{B}$ to stand for that of B. $P_{m_{1}}^{A}$ and $P_{m_{2}}^{A}$ were the largest two distribution probabilities of person A's neighbors and $P_{m_{1}}^{B}$ and $P_{m_{2}}^{B}$ were those of B's. There were four situations based on different $P_{m_{1}}^{A}, P_{m_{2}}^{A}, P_{m_{1}}^{B}$ and $P_{m_{2}}^{B}$ listed below. We used a constant $T h$ to represent the threshold of comparison.

(1) $P_{m_{1}}^{A}-P_{m_{2}}^{A} \geq T h$ and $P_{m_{1}}^{B}-P_{m_{2}}^{B} \geq T h$

In this case, we believed $C_{m_{1}}^{A}$ corresponding to $P_{m_{1}}^{A}$ and $C_{m_{1}}^{B}$ corresponding to $P_{m_{1}}^{B}$ were outstanding ones among those candidates. So we just chose $C_{m_{1}}^{A}$ as the emotion recognition result for person $\mathrm{A}$ and $C_{m_{1}}^{B}$ for person B.

(2) $P_{m_{1}}^{A}-P_{m_{2}}^{A}<T h$ and $P_{m_{1}}^{B}-P_{m_{2}}^{B} \geq T h$

In this case, $C_{m_{1}}^{B}$ corresponding to $P_{m_{1}}^{B}$ was selected as the recognition result of person B just as situation(1). But for person A, there wasn't such class with prominent performance, in other words, it was not sure which class should be 
selected as the result. In our system, we selected $C_{m_{i}}^{A}$ corresponding to $P_{m_{i}}^{A}$ defined in equation(2) as the recognition result for person $\mathrm{A}$.

$$
P_{m_{i}}^{A}=\arg \max _{i=1}^{2}\left(P_{m_{i}}^{A} \times\left(M_{A}\right)_{m_{i}^{A} m_{1}^{B}} \times P_{m_{1}}^{B}\right)
$$

Here, we embedded emotion interaction information mentioned above into the speech emotion recognition system. Such method could save those candidates in $\left\{C_{1}, C_{2}, \cdots, C_{M}\right\}$ which might have lower probabilities because of noise. As human beings, we also use this rule in emotion perception. If we are not sure about the other's emotional state, we'd like to judge it by our experience on what emotion would be most likely.

(3) $P_{m_{1}}^{A}-P_{m_{2}}^{A} \geq T h$ and $P_{m_{1}}^{B}-P_{m_{2}}^{B}<T h$

This case is similar to situation(2) excepting for using matrix $M_{B}$ instead of $M_{A}$. Here we omit the detailed operations.

(4) $P_{m_{1}}^{A}-P_{m_{2}}^{A}<T h$ and $P_{m_{1}}^{B}-P_{m_{2}}^{B}<T h$

In this case, both $\mathrm{A}$ and $\mathrm{B}$ could not find sure recognition results. When recognizing emotional state of person A, $C_{m_{i}}^{A}$ corresponding to $P_{m_{i}}^{A}$ defined in equation(3) was selected as the result.

$$
P_{m_{i}}^{A}=\arg \max _{i, j=1}^{2}\left(P_{m_{i}}^{A} \times\left(M_{A}\right)_{m_{i}^{A} m_{j}^{B}} \times P_{m_{j}}^{B}\right)
$$

Similarly, person B's recognition result depended on $P_{m_{j}}^{B}$ defined in equa$\operatorname{tion}(4)$.

$$
P_{m_{j}}^{B}=\arg \max _{i, j=1}^{2}\left(P_{m_{i}}^{A} \times\left(M_{B}\right)_{m_{i}^{A} m_{j}^{B}} \times P_{m_{j}}^{B}\right)
$$

\section{Experiment Result}

In our experiment, we set $k$ to 10 in K-Nearest-Neighbor searching and Threshold $T h$ to $20 \%$. In order to evaluate the performance of our new method, we
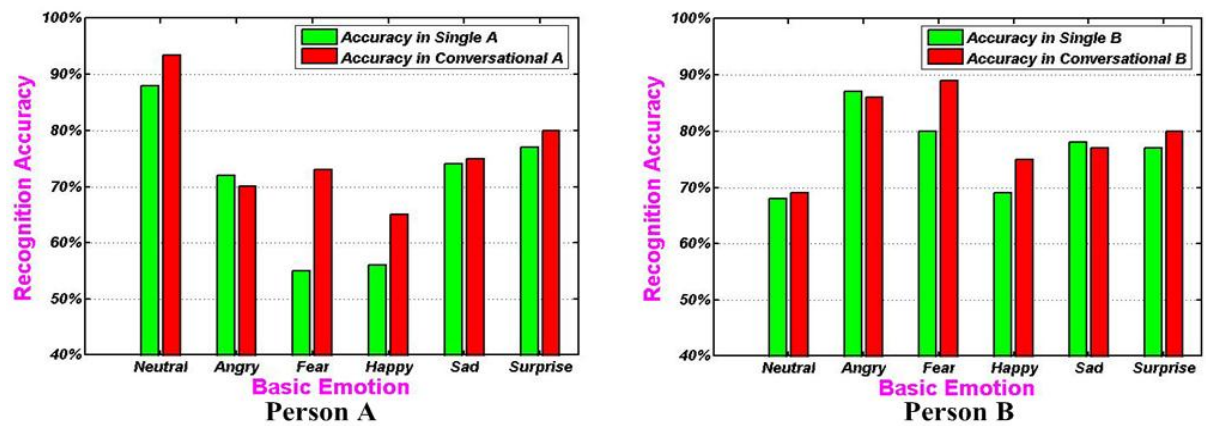

Fig. 2. Recognition Accuracy for person A and person B in our method (Accuracy in Conversational X) and traditional method (Accuracy in Single X) 


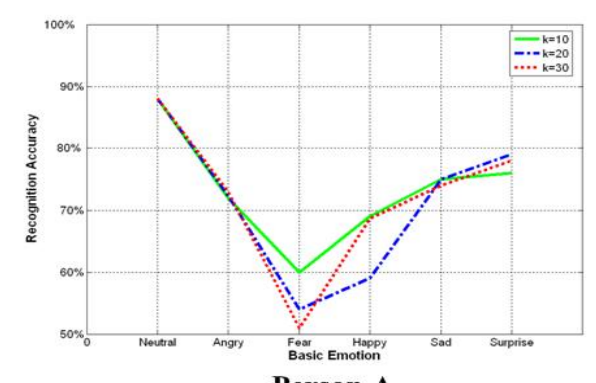

Person A

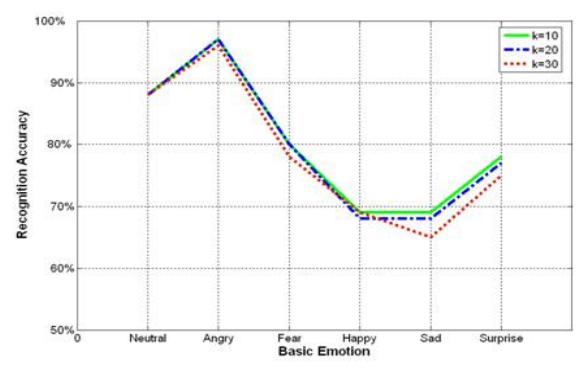

Person B

Fig. 3. Recognition Accuracy on Different Choice of $k$

also included a traditional emotion recognition process. In the traditional way, emotions of two persons in dialog were recognized separately and classical KNN classification was employed. The recognition accuracy of six emotions using our enhanced emotion recognition method and the traditional method is shown in Figure 2. From the figure, we can find out that the enhanced speech emotion recognition system outperforms traditional method on almost all of the basic emotions. On the average, our system is observed $5 \%$ relative improvement on person $\mathrm{A}$ and $3 \%$ on person $\mathrm{B}$ compared with traditional method. For person A, traditional method has different performance on different emotion. The accuracy on emotion fear is $30 \%$ lower than that on neutral, which will impact on the system performance. Our method balances the performance on each emotional state and improve the recognition accuracy totally.

Besides, the performance on different choice of $k$ in K-Nearest-Neighbor searching is compared in Figure 3. None of the $k$ achieves outstanding result compared with other choices. But $k=10$ always has acceptable performance, especially on the emotion fear of person A. In addition to performance, simple computation is also an advantage of $k=10$.

\section{Conclusion and Future Work}

This paper presents an enhanced speech emotion recognition system based on discourse information between human beings. Instead of hurriedly choosing one class as the recognition result, all possible classes were investigated. Experiment result shows that the enhanced method makes improvements at almost all of the emotional states and balanced the performance on every emotion. As we expected, interaction information used in the communication of humans did help the emotion recognition of computers.

The emotion interaction matrix we collected is only a beginning work. Because of the small conversation database, the correlation of emotional states between talking people can not be well indicated. More efforts should be put on the collection of conversation database and the discovery of emotional relationship between talking people. 


\section{Acknowledgement}

The work is partly supported by National Natural Science Foundation of China (60203013). And We thank Cheng Jin, Qi Wu and Weiguang Wang for their generous help to our experiment and paper.

\section{References}

1. A. Mehrabian, "Communication without words", Psychology Today, 2(4), pp. 5356,1968

2. Z. J. Chuang and C. H. Wu, "Emotion recognition from textual input using an emotional semantic network", in Proceedings of ICSLP, Denver, Colorado, USA, 2002, pp. 2033C2036.

3. B. Schuller, G. Rigoll and M. Lang, "Speech emotion recognition combining acoustic features and linguistic information in a hybrid support vector machinebelief network architecture ", in Proc. IEEE International Conference on Acoustics, Speech, and Signal Processing, Volume 1, pp. 577-580, May 2004.

4. D. Ververidis, C. Kotropoulos and I. Pitas, "Automatic emotional speech classification", in Proc. IEEE International Conference on Acoustics, Speech, and Signal Processing, Volume 1, pp. 593-596, May 2004.

5. C. Lee, S. Narayanan, and R. Pieraccini, "Recognition of negative emotions from the speech signal", in Proc. Automatic Speech Recognition Understanding, Dec. 2001.

6. J. Yuan, L. Shen, and F. Chen, "The acoustic realization of anger, fear, joy, and sadness in chinese", in Proceedings of ICSLP, Denver, Colorado, USA, 2002, pp. $2025 \mathrm{C} 2028$.

7. J. Liscombe, G. Riccardi and D. Hakkani-Tür, "Using Context to Improve Emotion Detection in Spoken Dialog Systems", In the Proceedings of EUROSPEECH'05, September, 2005.

8. J. Ang, R. Dhillon, A. Krupski, E. Shriberg, and A. Stolcke, "Prosody-based automatic detection of annoyance and frustration in human-computer dialog", in Proceedings of ICSLP, Denver, Colorado, USA, 2002, pp. 2037C 2039.

9. C. M. Lee and S. Narayanan, "Towards detecting emotions in spoken dialogs", IEEE Transactions on Speech and Audio Processing, in press, 2004.

10. A. Batliner, K. Fischer, R. Huber, J. Spilker, and E. Nöth, "How to find trouble in communication", Speech Communication, vol. 40, pp. 117C143, 2003.

11. D. Crystal, "Prosodic Systems and Intonation in English", Cambridge University Press, 1969

12. D. Crystal, "The English Tone of Voice", Edward Arnold, 1975

13. D. Crystal, "The Cambridge Encyclopaedia of the English Language", Cambridge University Press, 1995

14. M. You, C. Chen and J. Bu, "CHAD: A CHINESE AFFECTIVE DATABASE", in Proc. Affective Computing and Intelligent Interaction, pp. 542 - 549, 2005 